\title{
Lapurdum
}

Euskal ikerketen aldizkaria | Revue d'études basques |

Revista de estudios vascos | Basque studies review

$6 \mid 2001$

Numéro VI

Compte rendu de Anne-Marie Lagarde, L'univers psychique des Basques : une instauration de la symétrie des sexes. Expression sociale et linguistique

Thèse d'Etudes Basques U.P.P.A., 2000

\section{Manex Goyhenetche}

\section{OpenEdition}

\section{Journals}

Édition électronique

URL : http://journals.openedition.org/lapurdum/1252

DOI : 10.4000/lapurdum.1252

ISSN : 1965-0655

Éditeur

IKER

Édition imprimée

Date de publication : 1 octobre 2001

Pagination : 421-437

ISBN : 2-84127-156-0

ISSN : $1273-3830$

Référence électronique

Manex Goyhenetche, "Compte rendu de Anne-Marie Lagarde, L'univers psychique des Basques : une instauration de la symétrie des sexes. Expression sociale et linguistique », Lapurdum [En ligne], 6 | 2001, mis en ligne le 01 juin 2009, consulté le 29 janvier 2020. URL : http://journals.openedition.org/ lapurdum/1252 ; DOI : 10.4000/lapurdum.1252 


\section{Compte rendu de Anne-Marie Lagarde, L'univers psychique des Basques : une instauvation de la symétvie des sexes. Expression sociale et linguistique}

Thèse d'Etudes Basques U.P.P.A., 2000

Des thèmes similaires ont déjà attiré l'attention des auteurs et des chercheurs. Cette fois-ci, l'entreprise a l'intérêt de mobiliser les ressources de l'interdisciplinarité, dans un éventail très large et très varié des sciences humaines. Elle comporte des angles d'approche divers et différents. Certains chapitres, auxquels je me suis intéressé tout particulièrement, pourraient relever, dans une certaine mesure, de l'histoire ethnographique ou anthropologique ayant pour champ d'investigation le repérage des attitudes, des comportements, des traits de psychisme à partir des traces qui nous en ont été laissées (1). L'entreprise n'est pas à l'abri des risques déjà signalés par Ferdinand Braudel (2), notamment sur l'utilisation - ou la tentative de découverte - de la notion de "cultures primitives". La tâche n'est pas impossible, d'un point de vue historiographique, à condition que la méthode d'investigation repose sur l'enquête documentaire.

Aussi, la contribution ou le regard critique que je porte sur ce travail ne concerne que le domaine que je connais : l'histoire. Pour des raisons d'épistémologie, il n'est pas question d'aborder les domaines qui ne sont pas miens, mais que l'auteur aborde : philosophie, philologie, psychologie, droit, ethnologie, anthropologie, psychanalyse. Les notes qui suivent n'ont pas pour objet de nier l'importance et l'intérêt de ce travail, mais, dans une perspective historiographique, d'aider à la réflexion d'ordre épistémologique qu'il est nécessaire de mener autour d'une discipline appelée, ici, dans la thèse, "Etudes basques".

Je n'aborderai pas le sujet même du travail (l'univers psychique des Basques). Je me contenterai seulement de quelques remarques ou corrections, d'ordre historique ou historiographique, suscitées par la lecture linéaire du texte dactylographié de la thèse. 


\section{P. 27 : "l'euskara langue paléolithique" :}

Les conclusions des dernières recherches sur cette question sont très loin d'une telle affirmation. J.B. Orpustan, ouvrant les débats du Colloque du C.N.R.S. tenu à Bayonne, en 1993, évoquait le "scepticisme général qui s'est emparé de la bascologie moderne quant aux origines du basque" (3).

P. 29 : “L'examen balayera à grands traits le cours de l'histoire, chapitre après chapitre. Antiquité d'abord, avec une incursion dans la mythologie basque. Période allant de la pax romana au XIIème siècle, ensuite. Puis du XIIème au XVIII'̀me siècle. Enfin XIXème et XXème siècle”.

J'admire une telle capacité intellectuelle et épistémologique à pouvoir embrasser ainsi - et ramasser en quelques 130 pages dactylographiées - tous les temps de l'histoire des Basques de l'Antiquité à nos jours, alors que les historiens sont toujours réduits à se poser bien des questions, à poursuivre inlassablement la quête documentaire de masses d'archives non exploitées encore et où doivent se cacher bien des éléments d'éclairage de notre histoire.

\section{P. 30 : "Le regard de Strabon".}

Dans la thèse, la présentation historique des informations fournies par Strabon est très contestable: Strabon signale les Cantabri, les Berones, les Barduli, les Vascones, les Jacetani, les Ilergetes, les Celibères. Pour pouvoir en tirer des déductions d'ordre anthropologique et/ou ethnologique, voire sociologique sur "les ancêtres des Basques", il faudrait reprendre le traitement documentaire, à partir notamment des travaux de Koldo Larrañaga (Université du Pays Basque), peu connus car publiés en euskara (4).

P. 31 : “(...) témoigne de l'existence de tribus dont les noms rebutent Strabon : il s'agit donc pour lui sur le plan linguistique d'une expérience d'altérité absolue".

L'emploi du verbe "rebuter" est ici proche du jugement de valeur, d'autant plus anachronique que, en l'état actuel de la documentation, il semble que Strabon n'ait jamais parcouru le Pays Basque. Né en Cappadoce (actuelle Turquie), ayant séjourné à Alexandrie (importante ville possédant une riche bibliothèque), ayant ensuite parcouru l'Italie de l'Empire romain, il a travaillé à partir des sources bibliographiques de Rome. Quels documents, quels témoignages a-t-il utilisé? Comment, de qui a-t-il recueilli le terme "wascones"?

Par ailleurs, le regard porté par Strabon n'a pas de quoi nous surprendre, c'est celui d'un homme de civilisation grecque et romaine. On rejoint là le thème de "l'altérité et la barbarie" ou "la barbarie et la civilisation" inséré dans les nouveaux programmes de T.P.E. (travaux personnels encadrés) de 
l'Education Nationale pour les lycées. Et l'on sait que bien des tiraillements existent entre anthropologues, ethnologues et historiens sur le thème de "l'altérité absolue" appliquée aux civilisations dites primitives et dont on tente de redécouvrir l'état primitif. Dans le cas de l'univers psychique des Basques, il s'agit en quelque sorte de reconstituer l'état primitif ou d'origine et d'en suivre les vicissitudes à travers le temps.

\section{P. 32 : lignes 13-17, toujours sur Strabon.}

Il est surprenant que pour l'étude des Vascons de cette même époque (celle de Strabon),- "il y a dans les structures médiévales de ces derniers au Moyen-Age (...)"- l'auteur de la thèse ait recours au "droit basque tel qu'il est attesté au XIIe siècle". Je serais heureux de connaître ce droit basque du XII siècle en Labourd, Soule, Guipuscoa, etc. Nous avons, pour la Navarre (et l'Aragon), des fors locaux qui apparaissent à Jaca (1076), à Estella (1090), Puente la Reina (1095), Sanguesa (1122), le nouveau quartier de San Saturnino (ou Cernino) à. Pampelune (1129), fors accordés en premier lieu aux "Francos" et non aux autochtones navarrais! Ce nouveau modèle juridique fut étendu au Guipuzcoa seulement à partir de la fin du XII ${ }^{\text {siècle }}$ (Saint-Sébastien en 1180) (5).

P. 33 : "L'examen des témoignages de l'Antiquité sur la "gynécocratie" cantabro-pyrénéenne" (...) "Le matriarcat cantabre (...)".

Toujours la même question pour l'Antiquité : quelle source documentaire historique nous autorise à établir un lien entre le matriarcat cantabre et celui des Vascons ("les données antiques concernant le droit féminin chez les Vascons")?

P. 33, note 52 : description du festin “(...) le long de la paroi un siège construit avec le mur, où l'on prend place selon son âge et son rang".

Le témoignage de la documentation écrite est attesté par l'apport de l'archéologie qui a mis à jour les sites d'habitat urbain de cette époque. Mais il reste toujours la question de fond : cela concerne-t-il les Cantabres, les Ibères, les Vascons, les Celtibères? A moins de faire de tout ce "melting pot" d'avant la lettre "nos ancêtres les Vascons". Et cela nous amène à une autre piste de quête documentaire et de recherche : à l'époque de Strabon, dans l'espace compris, grosso modo, entre les Pyrénées et la rive droite de l'Ebre, quel était le fonds autochtone (vascon?), quels étaient les apports exogènes? De quels peuples, de quelles migrations? de quelle (s) période (s) témoignent les sources tant archéologiques que documentaires? De quels éléments (autochtones, exogènes, endogènes) était composée la civilisation dite "celti- 
bère" (si tant est qu'on puisse utiliser une telle appellation, mais les textes de Strabon nous y autorisent).

P. 41 "Le goût de la fête (...)" (...). "L'ensemble de ces notations n'est pas sans nous rappeler les "akelarre" (ou fêtes du bouc) qui avaient lieu les nuits de pleine lune, et furent attestés au début du XVIIe siècle encore en Pays Basque par de Lancre (...)".

Pour expliquer les us et coutumes des "ancêtres des Basques" dans l'Antiquité, avoir recours au traité de Pierre de Lancre au XVII' siècle, donne le vertige. La question des procès de sorcellerie, dont la grande période se situe entre la fin du XVe siècle et le XVIIe siècle, est assez bien circonscrite à l'heure actuelle par les historiens, même si des interrogations demeurent toujours (voir les travaux de Julio Caro Baroja, Bernard Guillemin, Nicole Jacques-Chaquin, ou les actes du Colloque International tenu à Fontenay Saint-Cloud en 1992) (6). Nicole Jacques-Chaquin et Maxime Préaud ont montré, dans la présentation des actes de ce colloque, que, un peu partout en Europe, à partir de la fin du XVe siècle, la cérémonie du sabbat a constitué l'élément essentiel de l'imaginaire de la sorcellerie. Il s'agit d'un récit que l'on (le juge, les autorités) fait faire à la victime, qui est obtenu par des méthodes de coercition et de mauvais traitements. Le stéréotype ou l'archétype du sabbat a été élaboré par les inquisiteurs et les démonologues (Pierre de Lancre en était un). A travers toute l'Europe (notamment en Suisse, en Allemagne, vallées italiennes, Aragon), nous avons les mêmes rites de la "lande au bouc" (akelarre).

$$
\text { P. } 42 \text { "Et le viol (...)". }
$$

Avoir recours à la Coutume de Soule de 1520 pour résoudre cette question, dans le cadre de l'étude de Strabon qui ne l'évoque même pas d'ailleurs, laisse perplexe en ce qui concerne le traitement historiographique. Les mesures contre le viol étaient prévues dans les législations médiévales, par exemple dans le titre III du livre quatre du Fuero General: "de fuerzas de mugeres et de adulterios"; chapitre III : "que pena ha el yfanzon que forza yfanzona (...)" ; chapitre IV : "que pena ha yfanzon que forza a villana"; chapitre VI : "que pena ha villano que forza a yfanzona"; chapitre VIII : "que pena ha qui forza a muyller casada"; chapitre IX : "que pena ha ombre casado que forza a muyller casada", etc.

P. 43 "Dispositions du droit basque concernant la guerre de conquête : elle est proscrite. Les Biscayens avaient l'obligation, de par le Fuero, de défendre leur terre, mais, arrivés aux limites de celle-ci, ils ne devaient pas poursuivre l'ennemi dans un esprit de conquête. Ils plantaient symboliquement leurs 
glaives dans "l'arbre de Malato" qui se trouvait à la frontière biscaïenne".

L'auteur tombe ici dans l'imprécision, l'anachronisme et le contre-sens historique. Tout d'abord, comme source documentaire de cette pratique médiévale, l'auteur cite Philippe Oyhamburu, de profession journaliste et artiste. Pour une thèse digne de ce nom, on aurait aimé avoir la référence historique précise, ainsi que le contenu exact de cette pratique. Il s'agit de la loi $\mathrm{V}$ du titre I du Fuero de Biscaye élaboré en 1526 (7) qui stipule : $1^{\circ}$ ) que les nobles de Biscaye (Los Caballeros-Escuder̃os, hombres Hijosdalgo), et non tous les Biscayens, ont l'obligation d'être au service du seigneur de Biscaye ("que a su servicio los mandase llamar"), sans recevoir de solde ("sin sueldo alguno"), jusqu'à l'arbre Malato ("el Arbol Malato"), situé à Lujando. Mais si le seigneur leur mande d'aller au-delà, il devra assurer la solde de deux mois, s'ils sortent jusqu'aux ports, "les debe pagar el sueldo de dos meses, si hubieren de ir a aquende los puertos", et, au-delà, trois mois. Si ces conditions ne sont pas remplies, les nobles biscayens ne sont pas tenus d'obéir à leur seigneur.

A vrai dire, une telle pratique est bien connue des historiens médiévistes : il s'agit de la version biscayenne du droit de ban (commandement), plus exactement de la "chevauchée" (service militaire dû par le vassal hors de la seigneurie du seigneur et dont la durée était limitée dans les coutumes), et du "service d'ost"; ce dernier était illimité dans la durée et l'espace, mais un complément de solde était versé par rapport aux gages de la "chevauchée" (8).

P. 48 "Quant à la prise en compte de sa parole (celle de la femme "vasco-cantabre"), elle apparaît dans un épisode conté par Plutarque et rapporté par Albert Tessier" (auteur d'un ouvrage publié en 1918).

Ici aussi, nous attendons en vain la source documentaire précise, la citation exacte de Plutarque, l'auteur se contentant de donner une source de deuxième main (celle d'Albert Tessier).

P. 48 “la ville gersoise d'Auch qui s'appelait autrefois Elliberre et avait été fondée par des tribus vasconnes".

Sur une telle fondation vasconne, nous n'avons aucune preuve documentaire écrite. Strabon situe les Ausci parmi les "Akouitanos". Il en est de même pour Pline "Aquitanicae sunt (...) Ausci (...)”, ainsi que pour Pomponius Mela ("Aquitanorum clarissimi sunt Ausci"). Ce dernier, qui vivait au Ier siècle après J.C., ajoute une indication non donnée par ses prédécesseurs : "urbes opulentissimae in Auscis Elimberrum" (9). Aucun auteur gréco- 
romain ne mentionne une fondation vasconne.

P. 65 "Au sujet de "L'Etxe (...) ce tout indivisible qui englobe la demeure ancestrale et ses terres, et la demeure des morts. Le culte rendu dans la maison s'adressait indubitablement aux âmes des ancêtres" (...) "Ce culte a conditionné jusqu'à aujourd'hui la vie économique et sociale des Basques et ce système juridique si original qui apparaît dans les fors et coutumes consignés par écrit à partir du XIVème siècle".

Ce paragraphe aussi contient bien des confusions. D'accord sur le fait que le régime de conservation et de succession des biens apparaît dans les textes législatifs à partir de la fin du Moyen-Age. Mais je ne vois pas dans quel document on évoque la notion de "demeure des morts" ou "le culte rendu (...) aux âmes des ancêtres." Et l'auteur elle-même ne donne aucune référence précise.

P. 71 "Après la conquête romaine" (...). L'on ne dispose d'aucune source concernant les aménagements précis effectués par les envahisseurs sur le plan administratif. Seul l'écho de la lutte est parvenu jusqu'à nous puis le silence de la "pax romana".

Ou n'est-ce pas plutôt que l'auteur ne connaît pas les sources? Il suffit de reprendre les travaux de transcription réalisés par Koldo Larrañaga (cité en note 4), notamment les chapitres II et III de son corpus documentaire : voilà un matériau de choix pour connaître, du moins quelque peu, le Pays Basque au temps de la "pax romana", sur le plan économique, démographique, social, administratif. Par ailleurs, Ies recherches archéologiques menées à Irun, Andelos, Pampelune, Gasteiz, Saint-Jean-le-Vieux, etc, permettent de jeter un éclairage partiel sur la période romaine en Pays basque.

P. 76 et suivantes: "Du Ve au Xe siècle".

Roger Collins et Eugène Groyheneche sont cités abondamment. Par contre, aucune mention des travaux de Michel Rouche et Renée Mussot-Goulard (10). Ces deux médiévistes, excellents latinistes, ont entièrement renouvelé la connaissance de cette période en ce qui concerne l'histoire des Vascons.

P. 78. Au sujet de l' "état vascon pratiquement indépendant" et "par la suite, il semble qu'il y ait eu deux duchés: celui d'Aquitaine et celui de Vasconie" :

Que de confusions! Pourtant, il suffisait de reprendre les travaux de Michel Rouche et Renée Mussot-Goulard, même si des zones de contradiction et d'incertitude subsistent entre les deux auteurs. 
P. 79 "Les conséquences politiques de Roncevaux furent selon les spécialistes la création du royaume de Pampelune".

De quel spécialiste d'histoire médiévale s'agit-il? Si l'on suit Michel Rouche ou Mussot-Goulard, ou même Jose Maria Lacarra (op. cit. en note 5), la coalition aquitano-vasconne (c'est le terme exact qu'il faudrait employer) fut vaincue en 781, le fils de Charlemagne, Louis le Pieux contrôla l'espace Bordeaux - Pampelune (où il résida même en 810-812). La nouvelle rébellion des Vascons n'intervint qu'à partir de 816, après la mort de Sanche-Loup.

P. 80. Au sujet de Sanche le Grand : "la réunion des sept provinces sous un même sceptre fut de courte durée".

Les royaumes et domaines de ce roi s'étendaient bien au-delà de l'actuel Zazpiak Bat.

P. 81 “L'on peut penser que l'importance de la femme dans la société basque a pu freiner la christianisation".

La problématique introduite par l'auteur est intéressante et l'hypothèse pertinente : encore faut-il apporter un minimum de matériau documentaire à l'appui de l'hypothèse, ce qui n'est pas le cas.

P. 85 "Leur adhésion extrêmement tardive à la royauté et au christianisme" (au sujet des Basques).

L'auteur est en contradiction avec ce qu'elle a écrit dans les pages précédentes au sujet de "la conversion au christianisme". Il est vrai que l'on trouve ici le thème récurrent de la conversion tardive des Basques au christianisme. Se cantonner au leitmotiv ne fait guère avancer la recherche si l'on ne s'attelle pas à l'étude des sources écrites, épigraphiques, à l'examen des fouilles archéologiques. Il serait temps là aussi, au cours d'un Colloque par exemple, d'en dresser le bilan (11). On verrait à ce moment-là, hypothèse légitime, que le processus d'implantation du christianisme en Pays Basque fut analogue à ce qui se passa ailleurs : la nouvelle religion se répandit, à partir du pourtour méditerranéen, le long des voies romaines, d'abord dans les noyaux urbains et les cités, ensuite dans le pagus, et progressivement dans le saltus vasconum. Dès le IVe siècle, Prudence, Vascon de la cité de Calagurris, en vallée de l'Ebre (Vasco Iberus), issu d'une famille chrétienne, déplore "la grossière gentilité des Vascons" (Vasconum gentilitas) d'alentour. Toujours au IVe siècle, Ausin et Paulin de Nole, deux Aquitains romanisés, chrétiens, expriment la crainte du "Vasconis saltus", mais en 626, d'après le Chronicon de Frédégaire, l'évêque d'Eauze fut condamné à l'exil pour complicité avec les Vascons (rebellione Wascorum) (11). 
P. 85 “Jamais l'organisation qui permit à ce peuple de s'opposer aux envahisseurs germaniques très supérieurs en nombre n'a été explorée ni n'a tout simplement suscité de questions, tant il est vrai que pour les historiens (...)".

Au risque de plagier Hubert Reeves, il est bon de rappeler que le discours scientifique n'est pas un discours d'affirmation d'une vérité, mais une recherche permanente, un questionnement. Avant d'affirmer, de façon péremptoire, que "jamais...", peut-être faut-il commencer par lire l'importante contribution de Michel Rouche sur ce sujet (cité en note 10).

P. 86 et 87 "Les fueros ou fors" pour l'étude de "l'organisation sociale des Basques".

Je suis entièrement d'accord sur le fait que l'histoire institutionnelle permet de saisir les traits de l'organisation sociale. Mais je dois avouer que la présentation historique qui en est faite contient énormément d'imprécisions et de confusions. Une abondante bibliographie existe sur la question, et l'auteur se contente de citer Roger Collins qui n'est pas du tout spécialiste en la matière. Aucune distinction entre les fors locaux ou municipaux et les fors généraux, étendus à un pays, une vallée; aucune référence aux Hermandades dont les ordonnances sont à l'origine de bien des fueros et des Juntas notamment en Guipuscoa, mais aussi en Alava; confusion entre le fuero d'Alava et d'Ayala; ignorance des fors et coutumes des versants septentrionaux (Soule, Labourd, Basse-Navarre) élaborés bien avant le XVIe siècle (coutume de Bayonne au XIIIe siècle, coutume de Montory au XIVe siècle, de Labastide au XIVe siècle, de Saint-Jean-Pied-de-Port au moins dès le XIII ${ }^{e}$ siècle, d'Ostabat vers les XII'-XII' siècles, ordonnances de Ultrapuertos de 1341 , coutume du pays de Mixe réformée par Amanieu d'Albret dès 1316, etc (12)). Et le comble est atteint avec cette affabulation : "d'ailleurs, selon Roger Collins ce sont des colons vascons qui impulsèrent l'émergence des fueros de Castille".

P. 23 : “la version qu'en donne le vieux droit basque en ses coutumes transcrites à partir du XIVe siècle".

Une confirmation de plus sur les confusions qu'entretient l'auteur de la thèse sur les différentes époques d'élaboration, de confection, de transcription ou de rédaction nouvelle des fors et coutumes.

En guise de "vieux droit basque", il eût été intéressant, par exemple, de trouver dans cette thèse des développements sur les titres I, II, et III du Livre IV du Fuero General de Navarre consacrés respectivement au mariage (de 
casamientos), aux arras (donation antiphernale ou don du matin accordé par l'homme comme le prix de la virginité de la femme), aux viols, adultères et rapts (de fuerzas de mugeres et de adulterios), aux enfants (de criar fijos).

P. 87 "Inexistence de toute classe servile" (pour le Moyen-Age en Pays Basque).

Et que faire alors de la masse de documents d'archives du Moyen-Age où apparaissent les "pecheros", les "collazos", les "botoys", les "mesquinos", les "servi"? Que faire du Fuero General de Navarre où apparaissent des catégories paysannes sur lesquelles pesaient des redevances en nature ou en force de travail ayant nom cena ou albergada, peyta de fonsadera, pecha de reconoscienza, semana peon, labor ou facendera, pecha de perga, pecha de cazadores, etc, etc? (13). Certes, il ne s'agit pas de "classes" au sens où l'entendent les auteurs des $\mathrm{XIX}^{\mathrm{e}} \mathrm{XIX}^{\mathrm{e}}$ siècles, mais ce ne sont pas moins des catégories sociales inférieures.

P. 128 : "obedezco pero no cumplo" (...) ingénieuses figures juridiques lui permettant de se jouer des décrets madrilènes".

Ici aussi, comme pour el Arbol Malato, la référence documentaire n'est guère donnée, le contexte historique n’est pas dressé avec précision. Il s'agit du "pase foral". Les dernières études (14) montrent que cette pratique était l'équivalent du droit de remontrance usité par les parlements et les assemblées d'états à l'égard de toute mesure du souverain. Ce mécanisme de contrôle des décisions royales trouve son origine dans le droit castillan du bas Moyen Age. Il ne fut introduit en Biscaye, Guipuscoa et Navarre qu'aux XVe-XVIe siècles. On ne le trouve pas dans les provinces basques septentrionales.

P. 200 : "Présentation des Cagots".

Francisque-Michel est abondamment cité. Mais rien sur les recherches d'universitaires actuelles comme Véronique Bériac ou Benoit Cursente (15) qui ont renouvelé la problématique de ce sujet.

P. 246. Au sujet de la délégation de "tous les Vascons seniores" qui se rendit au VIIe siècle auprès du roi Dagobert : “Il n'y a aucune raison de penser que la perception du chroniqueur Frédégaire soit fausse et que les "seniores" ne soient des chefs de parentèle (...). Ceci n'enlève rien de sa réalité au fait que par ailleurs, dans l'ensemble de la société vasconne, le système à maison prévalait depuis très longtemps (...)" et sûrement au temps de Strabon". 
Effectivement, il n'y a aucune raison d'opposer système de parentèle et système à maison. Mais dans le cadre de la problématique étudiée dans la thèse, il serait intéressant de poser la question suivante : dans le contexte du VIIe siècle, et plus exactement celui des VIe-VIIIe siècles, marqués par ce que Georges Duby appelle la "civilisation de la guerre et de l'agression (...) née des grandes migrations" (16), qu'était un "senior" vascon? Quelle évolution, par rapport à "la société traditionnelle basque" (voir p. 12 de la thèse) entraînèrent ces trois siècles de guerre? Dans quelle mesure ceux-ci intervinrent-ils dans "la genèse du lignage patrilinéaire" (17)? On peut prolonger le questionnement à partir du fait qu'à la fin du VIIIe siècle, selon les chroniqueurs francs, les Vascons vaincus furent décimés, massacrés, les chefs ou princes (les "seniores" de Frédégaire?) qui étaient vivants, pris en otage avec les enfants. Dans l'organisation (ou la réorganisation) sociale, quelle transformation, adaptation subirent, par voie de conséquence, les femmes vasconnes?

On peut poursuivre cete interrogation, l'étendre vers d'autres périodes charnières. La lecture de certains documents navarrais du haut Moyen Age (voir Documentacion medieval de Leire et Coleccion diplomatica de Irache cit. en note 13) mettent en lumière, par endroits, l'existence de structures sociales et familiales servant de réserve de main-d'œuvre. Dans ces documents, l'emploi fréquent de termes comme servus, servi, servire (ou d'expressions analogues) montre que le premier devoir est le service. La valeur sociale et économique de la famille semblait, dans ce cas, organisée autour de la gestion des redevances, des perceptions et des réquisitions.

Par contre, les XI'-XIII dental, furent caractérisés en Pays Basque par l'émergence et l'expansion des fors et coutumes. Dans le cadre de la renaissance du commerce et de l'essor des villes et des foires, notamment sur la route de Saint-Jacques-deCompostelle, les nouveaux noyaux de population, acquirent le droit de se constituer en corps juridique et administratif autonome, en "commune". Les relations sociales furent altérées, les liens avec les seigneurs, lä̈ques ou ecclésiastiques, se distendirent. Dans ce contexte, quelle évolution ou transformation connut la femme quant à sa place et à son rôle ? La valeur sociale et économique de la famille fut-elle la même que dans les périodes antérieures? 
Il s'agit donc de repérer, dans le temps, les permanences et les mutations du "système à maison" (pour reprendre l'expression de l'auteur), du modèle familial qui a été construit, ici comme ailleurs, par la culture (ou les apports des différents substrats de culture) et par l'histoire.

P. 247 : Au sujet de l'analyse du Prologue du Fuero General de Navarre par Manex Goyhenetche (Histoire générale du Pays Basque, T. I) : "L'on comprend et l'on partage le souci qui sous-tend son argumentation : car il s'agit d'éviter le péril d'une idéologie raciste (...)".

Mais non! Je ne vois pas ce que viendrait faire l'idéologie dans une analyse ou un commentaire de document historique. Comme on l'apprend aux lycéens pour la préparation au baccalauréat, l'analyse d'un document commence par l'étude de la nature de ce document, l'origine de son inspiration, sa source, les conditions de sa rédaction, l'identité de ses rédacteurs. C'est ce que j'ai essayé de faire, tout comme pour les autres documents historiques.

Pour revenir au Fuero General, à l'heure actuelle, bien des historiens sont convaincus que la rédaction du Prologue fut une entreprise de mystification impulsée ou inspirée par l'historien navarrais du XIII siècle, Rodrigo Ximenez de Rada. La chose était courante au Moyen Age comme l'a démontré Julio Caro Baroja (18).

Par ailleurs, il n'y a rien de surprenant qu'à l'époque de la rédaction du Fuero General, la pratique juridique en Navarre (et non la "pratique judiciaire" comme l'écrit l'auteur de la thèse) s'inspire des traditions et des enseignements juridiques de Rorne, de Lombardie, de France. La Navarre ne constituait pas un îlot. II faut placer la rédaction du Fuero General dans le contexte du XII' siècle empreint d'une forte influence juridique impulsée notamment par l'école de Bologne. Il faut savoir que le prestige juridique de Bologne était à son apogée à cette époque, en Occident, et notamment dans les royaumes dits ibériques, dont faisait partie la Navarre (19).

P. 252 : au sujet de l'interdiction faite à la femme au Biltzar de Labourd en 1784 et mentionnée par Manex Goyhenetche dans Histoire générale du Pays Basque (T. II, p. 265) : “L'on s'attend donc à ce qu'il explique la raison de l'interdiction (...) du moins à ce qu'il pose la question." (...) . L'explication concernant l'interdiction (...) ne viendra pas".

L'auteur aurait dû lire la page 266 où je pose la question avant de proposer une recherche : "Le vent de la contestation féminine soufflait-il dans les campagnes labourdines? La piste reste ouverte pour l'investigation" (Histoire générale du Pays Basque, T. II, p. 266). 
P. 253 : "Il (Manex Goyhenetche) est convaincu que seul le Siècle des Lumières apportera la touche manquant à la conception de la démocratie".

A l'appui d'une telle affirmation à mon sujet, l'auteur avance une interview donnée au journal Enbata. Tout le monde sait que les interviews sont rarement contrôlées par celui qui les donne et ne constituent pas une position de thèse. Par ailleurs, il serait bien illusoire, et surtout anachronique, d'appréhender le fonctionnement des institutions de l'Ancien Régime en terme de "démocratie" dont le concept apparaît tardivement (époque de Bossuet). Les institutions de l'Ancien Régime furent bâties sur des textes normatifs qui encadraient le réel, assuraient la permanence de certains comportements sociaux, économiques, moraux, politiques, culturels, donc prétendaient régler les rapports sociaux dans leur ensemble. Cela, dans un temps donné, dans un espace déterminé. L'objet de l'historien, dans ce cas précis, est d'essayer d'établir un lien entre l'histoire institutionnelle et l'histoire sociale. C'est modeste et ambitieux à la fois. Des historiens tentent de le faire en Pays Basque (20).

P. 252-254: Au sujet de Manex Goyhenetche (Histoire Générale du Pays Basque, T. II) : "On a à lire les développements de l'historien l'impression de naviguer entre deux plans de réflexion, voire dans un malentendu, et la question sur laquelle on reste, tant on est dérouté parfois par sa façon de présenter les choses (....). Et l'on reste sur sa faim".

Mais je crois que le malentendu est total, car il n'a jamais été dans mes objectifs et mes propos d'aborder, même d'un point de vue historiographique, la question du matriarcat chez les Basques, mais, dans une perspective d'histoire institutionnelle et sociale, de jeter les jalons d'une investigation sur le rôle et la place de la femme, dans le sillage de ce que propose Jose Antonio Azpiazu, position admirablement ramassée dans le titre : "Soumission et pouvoir" (21). Et la réalité sociale historique est souvent difficile à saisir, compliquée, contrastée, échappe à la simplification, à nos schémas culturels et idéologiques. Pour l'historien, elle exige l'inventaire des sources et le traitement documentaire, sans écarter les sources orales, sans minimiser l'apport de l'anthropologie sociale et historique, l'apport de l'histoire des mentalités.

P. 249 : "Il (Manex Goyhenetche) ne partage pas la conviction de certains chercheurs du domaine des études basques quant à une égalité structurelle des sexes dans l'histoire de ce pays. Il s'en tient à tout ce qui prouve la dépendance des femmes dans le Fuero General de Navarre".

L'égalité (structurelle ou pas) des sexes ne se trouve pas dans une histoire intemporelle, engendrée par nos chimères et nos imaginations, mais dans 
un réel complexe, situé dans un temps précis de l'histoire, dans un espace donné. Pour essayer de saisir ce réel complexe, autant que faire se peut, la quête documentaire est une démarche nécessaire, même si le document ne dévoile pas tout le réel.

Pour revenir au Fuero general, base du droit navarrais, l'auteur semble ne l'avoir pas consulté directement dans le texte. Pourtant, ce texte normatif qui devait encadrer le réel, est très riche d'informations sur le rôle et la place de la femme, sa capacité juridique, sur le contrat de mariage, la constitution des arras, le régime matrimonial, la condition de la femme pendant le mariage, les tâches de la femme selon que le mari était présent ou absent, les obligations réciproques des époux, l'administration des biens, la condition de la femme après la dissolution du mariage, les secondes noces, l'usufruit des veuves, etc. Faut-il faire abstraction de tous ces textes? Ne constituent-ils pas un matériau pour l'approche de l'univers psychique des Basques et la symétrie des sexes? Mais à un moment précis de leur histoire, la Navarre au XIII siècle. Surtout ne séparons pas la Navarre et le Pays Basque, et par voie de conséquence un quelconque droit basque que l'on ne trouverait pas dans le Fuero General. Certes, il est vrai que peu consultent le texte dans son ensemble, surtout côté Pays Basque Nord, car il est rédigé en navarro-aragonais et n'a pas été traduit en français (ni en basque non plus d'ailleurs). Pourtant, il reste un document fondamental pour connaître la société navarraise, celle des Basques, du XIII siècle. Le seul travail d'investigation réalisé de ce côté-ci a été mené par G. Desdevises du Dézert dont la thèse au titre évocateur ( De conditione mulierum....) mériterait d'être rééditée pour qu'on ne continue pas à faire une histoire intemporelle du droit basque (22).

Qu'on le veuille ou non, pour connaître l'histoire du Pays Basque, y compris celle de la femme en Pays Basque, la consultation approfondie du Fuero General est indispensable. C'est la Navarre aussi qui a conservé le plus de sources documentaires écrites, car elle constituait un royaume, et à ce titre disposait d'une administration permettant la conservation des archives. Cela n'a pas été pour d'autres territoires du Pays Basque.

Elargissons le débat, et plaçons-nous au carrefour de l'interdisciplinarité, entre l'histoire, l'anthropologie, voire la philosophie. Dans cette étude, en quête de l'univers psychique des Basques et de la symétrie des sexes, différents temps de l'histoire ont été évoqués : l'Antiquité, celle des Ibères comme celle de Strabon, ou celle de Rome; le temps de l'implantation du christianisme, celui de l'émergence et du renforcement des différents états (Navarre, France, Castille, Angleterre), les XIe-XII siècles des premiers fors locaux, le 
XIIre siècle du Fuero General, le XVIe siècle de la Coutume de Soule, le XVIII ${ }^{e}$ siècle du Biltzar de Labourd, la Révolution etc.

Tous ces temps (ces événements) constituent-ils les vicissitudes de l'histoire, les éléments d'altération par rapport à un état de nature originel, creuset en quelque sorte de l'identité basque, de l'être basque? Mais à ce moment-là, ne sommes-nous pas confrontés à la question de la nature et de l'histoire, dans la mesure où l'homme n'existe pas seulement à l'état de nature, il est aussi histoire (être historique), avec sa capacité à transformer et à se transformer, à devenir? Peut-on conjuguer l'être basque en faisant abstraction de l'événement et du devenir?

Car il n'y a pas de nature, d'état de nature, d'état primitif à l'état zéro. Les Basques, tout comme leurs ancêtres (si tant est qu'il faille chercher des ancêtres, et lesquels?), n'ont existé, comme tous les peuples, que dans l'événement (provoqué ou subi), qui les fait rentrer dans l'historique, plus exactement dans la gangue de l'histoire. Nier cela, c'est dépouiller de leur histoire les hommes et les femmes du Pays Basque.

Dans cette perspective, on peut considérer comme un modèle d'investigation les actes du Séminaire I.P.E.S. Ikastaroak tenu à Bilbao en 1996 (23). Nous avons là les prémices de ce qui pourrait être une histoire des femmes en Pays Basque. Sous l'égide de l'Université du Pays Basque ou de la Société Eusko Ikaskuntza, d'autres travaux de monographie, menés par de nouvelles équiipes de recherche, ont vu le jour à Bilbao, Vitoria, Bermeo (24).

L'univers des Basques qui s'en dégage, surtout celui des femmes, n'est plus ce que José Urrutikoetxea Lizarraga appelle "la grande essence dans un monde des essences" (25). Loin des procédés d'idéalisation, le traitement des sources documentaires restitue plütôt les images contrastées d'identités sociales et culturelles vécues, produites, forgées, moulées dans l'expérience de situations, d'histoires, d'existences historiques réelles. L'étude de la famille, vacille souvent, il est vrai, entre "les chemins tortueux de l'idéalisation idéologique" (26) et l'approche difficile des réalités historiques concrètes. C'est dans cette dernière voie que s'est engagée désormais l'historiographie en Pays Basque.

Manex Goyhenetche 


\section{NOTES}

1. Voir Michel Vovelle, De la cave au grenier, Québec, Serge Fleury, 1980.

2. Ferdinand Braudel, Ecrits sur l'Histoire, Flammarion, 1996. Les problèmes méthodologiques ont été aussi abordés par Christian Desplat, "Anthropologie et histoire", Charivaris en Gascogne. La "morale" des peuples du XVIe au XXe siècle, Berger-Levrault, 1982, p. 251-256.

3. (Sous la direction de J.B. Orpustan), La langue basque parmi les autres. Influences et comparaisons. Izpegi, 1994, p. 8.

4. Koldo Larrañaga Elorza, Euskal Herria Antzinatean. Materiale eta Agiriak, Kriselu, UNED, Bergara, 1988.

5. Voir les travaux de J.M. Lacarra, guère utilisés dans cette thèse, notamment la série Fueros de Navarra, Fueros derivados de Jaca, 1. Estella-San Sebastian, 1969, 2. Pamplona, 1975. On peut aussi consulter avec profit l'ouvrage de Marcelin Defourneaux, Les Français en Espagne aux XIe et XII ${ }^{e}$ s., P.U.F., 1947

6. Nicole Jacques-Chaquin et Maxime Préaud, Le sabbat des sorciers en Europe. XVe-XVIIle siècles. Jérôme Millon, 1993.

7. Texte consulté : El fuero, privilegios, franquezas y libertades del M.N. y M.L. señorio de Vizcaya, Bilbao, 1977.

8. Bibliographie à consulter : René Fédou, Lexique historique du Moyen Age, Colin, 1980. Jean Favier, Dictionnaire de la France médiévale, Fayard, 1993.

9. Pour l'ensemble des citations, voir K. Larrañaga, op. cit. en note 4.

10. Michel Rouche, L'Aquitaine, Jean Touzot, 1979, Renée Mussot-Goulard, Les princes de Gascogne, 768-1070, C.T.R., 1982.

11. Pour les références des sources bibliographiques, consulter Manex Goyhenetche, Histoire générale du Pays Basque, Elkarlanean, 1998, T. I, p. 130-135, 156-157.

12. Consulter Jornadas sobre cortes, juntas y parlamentos del pueblo vasco, Cuadernos de seccion Derecho, Eusko Ikaskuntza, 1984. Instituciones, economia y sociedad (siglos VIII-XV), Congreso de Historia de Euskal Herria, 1988, T. II. Antonio Beristain, Maria Angeles Larrea, Rafael Maria Mieza, "Fuentes de derecho penal vasco (siglos XI-XVI)", Anthropologia vasca, Bilbao, 1980, T. V.

13 Outre le Fuero General (édition de 1869 établie par Pablo Ilarregui et Segundo Lapuerta), bibliographie complémentaire : G. Desdevises du Dézert, "De la condition de la femme mariée en Navarre d'après le Fuero General", Revue des Pyrénées, année 1890, t. II, p. 804-833 (voir supra note 21), Maurice Berthe, Famines et épidémies dans les campagnes navarraises à la fin du Moyen Age, Paris, S.F.I.E.D., 1984, Angel J. Martin Duque, Documentacion medieval de Leire (siglos IX a XII), Pampelune 1983, Jose Maria Lacarra et Angel Martin Duque, Coleccion diplomatica de Irache, Pampelune, 1986. Comme matériau de choix susceptible d'être utilisé aussi bien par les historiens, les anthropologues que les ethnologues, signalons l'importante collection Fuentes Documentales Medievales del Pais Vasco, sous l'égide d'Eusko Ikaskuntza. 
14 Bibliographie à consulter : Ricardo Gomez Rivero, "Un derecho historico no. actualizado : el uso ou pase foral", Congreso de Historia del Pais Vasco, op. cit., p. 73-84. Du même auteur, El Pase foral en Guipuzcoa en el siglo XVIII, Sans Sebastian, 1982.

15 Françoise Bériac, Des lépreux aux Cagots. Une société marginale. Bordeaux, F.H.S.O., 1990. Benoît Cursente, "Hypothèses nouvelles sur un vieux problème : l'origine des cagots pyrénéens", Actes du Colloque international de Foix, 1998.

16. Georges Duby, Guerriers et paysans, Flammarion, p. 60.

17 Martin Aurell, La noblesse en Occident (Ve-XVe siècles), Colin, 1996, p. 63-64.

18. Julio Caro Baroja, Las falsificaciones de la Historia, Seix Barral. Biblioteca Breve, 1992. En fait, le thème de la mystification en histoire et surtout dans les chroniques médiévales, y compris celles concernant l'histoire des territoires basques, avait été déjà abordé par Ramon Menendez Pidal dans sa collection Catalogo de cronicas.

19. Bibliographie à consulter sur ce sujet et que semble ignorer l'auteur : Hortensia Viñes Rueda, Hablar Navarro en el Fuero general, Pampelune, éd. Gomez, 1977. J. Beneito, Fuero, costumbre y doctrina en el Derecho Medieval español, Madrid, 1968, p. 8, A. D'Ors, Nueva Enciclopedia Juridica, Barcelona, 1969.

20. Dans cette perspective, voir les nouveaux travaux de recherche menés par Susana Truchuelo Garcia, La representacion de las corporaciones locales guipuzcoanas en el entramado politico provincial, Alfonso de Otazu, El igualitarismo vasco: mito y realidad, Txertoa, 1986, Fernando Martinez Rueda, Los Poderes Locales en Vizcaya, U.P.V., Bilbao, 1994, Alfonso F. Gonzalez, Instituciones y sociedad guipuzcoanas en los comienzos del centralismo (1680-1730), Donostia-San Sebastian, 1995.

21. Jose Antonio Azpiazu, Mujeres vascas. Sumision y poder, Haranburu, 1995.

22 Georges Nicolas Desdevizes Du Désert, De conditione mulierum juxta forum Navarrensium, thèse de la Faculté des Lettres de Paris, Caen H. Delesques, 1888. Traduction en français, De la condition de la femme mariée en Navarre d'après le Fuero General, Toulouse, Privat, 1890. Extraits dans la Revue des Pyrénées, 1890, T. II, p. 804-833, la revue Euskal Erria, 1891, XXIV, p. 326-355, 366-372, 402-404.

23 Las mujeres vascas en la historia, I.P.E.S., Cuadernos de formacion, 24. Consulter aussi le $n^{\circ} 12$ : Emakumea Euskalerriko historian (La femme dans l'histoire du Pays Basque).

24. M. Arbaiza Villalonga, Familia, irabajo y reproducion social. Una perspectiva microhistórica de la sociedad vizcaina del Antiguo Regimen. U.P.V., Bilbao, 1996. Paloma Manzanos Arreal, "La mujer y el mundo del trabajo en la Vitoria del siglo XVIII", Revue Vasconia, n³ 30, année 2000, p. 397-411. Fernandez Fonseca, Maria Jesus Prado Antunez, Ana Isabel, "El trabajo femenino en la Bizkaia del siglo XIX : analisis del trabajo femenino en la villa de Bermeo en 1860", Ibidem, p. 413-427. Rocio Garcia Abad, "Mercado de Trabajo y Estrategias Familiares en las mujeres durante la primera industrializacion vizcaina : el hospedaje", V. Jornadas de Estudios Historico-locales. La familia en Euskal Herria, revue Vasconia, n² 28, p. 93-115. 
25 "Estamos ante la gran esencia en un mundo de las esencias", José Urrutikoetxea Lizarraga, "Ets-adi"/"Etse" ("Etxe")- "Familia"/ "Casa" : a los terrenos de la historia por los vericuetos de la idealizacion y la ideologizacion", V Jornadas de estudios historico-locales. La familia en Euskal Herria, op. cit., p. 278.

26 "Familia Vasca : ideologia y realidad : nuevas perspectivas" (Ibidem). 